\title{
State Complexity Investigations on Commutative Languages - The Upward and Downward Closure, Commutative Aperiodic and Commutative Group Languages
}

\author{
Stefan Hoffmann ${ }^{[0000-0002-7866-075 X]}$ \\ Informatikwissenschaften, FB IV, Universität Trier, Universitätsring 15, 54296 Trier, \\ Germany, hoffmanns@informatik.uni-trier.de
}

\begin{abstract}
We investigate the state complexity of the upward and downward closure and interior operations on commutative regular languages. Then, we systematically study the state complexity of these operations and of the shuffle operation on commutative group languages and commutative aperiodic (or star-free) languages.
\end{abstract}

Keywords: finite automata $\cdot$ state complexity $\cdot$ shuffle $\cdot$ upward and downward closure $\cdot$ commutative languages $\cdot$ group languages $\cdot$ aperiodic languages $\cdot$ star-free languages

\section{Introduction}

The state complexity, as used here, of a regular language $L$ is the minimal number of states needed in a complete deterministic automaton recognizing $L$. The state complexity of an operation on regular languages is the greatest state complexity of the result of this operation as a function of the (maximal) state complexities of its arguments.

Investigating the state complexity of the result of a regularity-preserving operation on regular languages, see [7] for a survey, was first initiated by Maslov in [21] and systematically started by Yu, Zhuang \& Salomaa in [31.

A language is called commutative, if for any word in the language, every permutation of this word is also in the language. The class of commutative automata, which recognize commutative regular languages, was introduced in $[\underline{3}$.

The shuffle operation has been introduced to understand the semantics of parallel programs [422 28/30]. The shuffle operation is regularity-preserving on all regular languages. The state complexity of the shuffle operation in the general cases was investigated in 1 for complete deterministic automata and in [5] for incomplete deterministic automata. The bound $2^{n m-1}+2^{(m-1)(n-1)}\left(2^{m-1}-\right.$ 1) $\left(2^{n-1}-1\right)$ was obtained in the former case, which is not known to be tight in case of complete automata, and the tight bound $2^{n m}-1$ in the latter case.

A word is a (scattered) subsequence of another word, if it can be obtained from the latter word by deleting letters. This gives a partial order, and the upward and downward closure and interior operations, denoted by $\uparrow U, \downarrow U, \Omega U$ and 
$\circlearrowleft U$, refer to this partial order. Languages that result from upward closure operation are also known as shuffle ideals. The state complexity of these operations was investigated in 9 10:12:19:24]

In 14 15]16]17 the state complexity of these operations was investigated in the case of commutative regular languages. The results are summarized in Table 1

\begin{tabular}{|c|c|c|c|}
\hline Operation & Upper Bound & Lower Bound & Reference \\
\hline$\pi_{\Gamma}(U), \Gamma \subseteq \Sigma$ & $n$ & $n$ & 1417 \\
\hline$U ш V$ & $\min \left\{(2 n m)^{|\Sigma|}, f(n, m)\right\}$ & $\mathrm{nm}$ & \begin{tabular}{ll|l|l|l|l|}
1 & 14 & 15 \\
\end{tabular} \\
\hline$\uparrow U$ & $\min \left\{n^{|\Sigma|}, 2^{n-2}+1\right\}$ & & Thm. 10] \& $12|17| 19$ \\
\hline$\downarrow U$ & $\min \left\{n^{|\Sigma|}, 2^{n-1}\right\}$ & $n$ & Thm. $10 \& 1719$ \\
\hline$\Omega U$ & $\min \left\{n^{|\Sigma|}, 2^{n-2}+1\right\}$ & $\left.\frac{n}{\Sigma \mid}\right)^{\mid 2}$ & Thm. 10 \& 17/19 \\
\hline$\circlearrowleft U$ & $\min \left\{n^{|\Sigma|}, 2^{n-1}\right\}$ & & Thm. 10 \& 17,19] \\
\hline$U \cup V, U \cap V$ & $n m$ & sharp, for any $\Sigma$ & [14]17] \\
\hline
\end{tabular}

Table 1. State complexity results on commutative regular languages, where $n$ and $m$ denote the state complexities of the input languages. Also, $f(n, m)=2^{n m-1}+$ $2^{(m-1)(n-1)}\left(2^{m-1}-1\right)\left(2^{n-1}-1\right)$ is the general bound for shuffle from [1].

A group language is a language recognizable by an automaton where every letter induces a permutation of the state set. The investigation of the state complexity of these languages was started recently [18.

A star-free language is a language which can be written with an extended regular expression, i.e., an expression involving concatenation, the Boolean operations and Kleene star, without using the Kleene star 23. The class of star-free languages coincides with the class of aperiodic languages [29], i.e., those languages recognizable by automata such that no subset of states is permuted by a word.

So, in this sense the aperiodic languages are as far away from the group languages as possible. In [14 15] it has been shown that every commutative and regular language can be decomposed into commutative aperiodic and commutative group languages in the following way.

Theorem 1 ([14 15]). Suppose $L \subseteq \Sigma^{*}$ is commutative and regular. Then, $L$ is a finite union of languages of the form $U \amalg V$, where $U$ is a commutative aperiodic language and $V$ is a commutative group language over a subalphabe 1 of $\Sigma$.

Here, we will investigate the state complexity of operations considered in [14 15 for general commutative regular languages for the commutative group and the

\footnotetext{
${ }^{1}$ Over the whole alphabet $\Sigma$, these languages are precisely the languages recognizable by automata whose transition monoids are 0-groups, i.e., groups with a zero element adjoined.
} 


\begin{tabular}{|c|c|c|c|c|c|}
\hline & \multicolumn{2}{|c|}{ Group Case } & \multicolumn{2}{|c|}{ Aperiodic Case } & \\
\hline Op. & $\leqslant$ & $\geqslant$ & $\leqslant$ & $\geqslant$ & Reference \\
\hline$\pi_{\Gamma}(U)$ & $n$ & $n$ & $n$ & $n$ & 17 \\
\hline$U \sqcup V$ & $(n m)^{|\Sigma|}$ & $n m$ & $(n+m-1)^{|\Sigma|}$ & $\begin{array}{l}\Omega(n m) \quad \text { if }|\Sigma|>1 \\
n+m-1 \text { if }|\Sigma|=1\end{array}$ & $\begin{array}{l}\text { Thm. } 13 \\
\text { Prop. 14 } \\
214 \text { 15] \& } \\
\text { Prop. 20 }\end{array}$ \\
\hline$\uparrow U$ & $n^{|\Sigma|}$ & $\mathrm{n}$ & $\min \left\{n^{|\Sigma|}, 2^{n-2}+1\right\}$ & $\Omega\left(\left(\frac{n}{|\Sigma|}\right)^{|\Sigma|}\right)$ & $\begin{array}{l}\text { Thm. 10, } \\
\text { Prop. 16, } \\
\text { Prop. 5] \& 19] }\end{array}$ \\
\hline$\downarrow U$ & 1 & 1 & $\min \left\{n^{|\Sigma|}, 2^{n-1}\right\}$ & $\mathrm{n}$ & $\begin{array}{l}\text { Thm. 10, } \\
\text { Prop. 15, } \\
\text { Prop. 19 \& 19 }\end{array}$ \\
\hline$\Omega U$ & $n^{|\Sigma|}$ & $n$ & $\min \left\{n^{|\Sigma|}, 2^{n-2}+1\right\}$ & $\Omega\left(\left(\frac{n}{|\Sigma|}\right)^{|\Sigma|}\right)$ & Eq. (1) \\
\hline$\circlearrowleft U$ & 1 & 1 & $\min \left\{n^{|\Sigma|}, 2^{n-1}\right\}$ & $n$ & Eq. (1) \\
\hline $\begin{array}{l}U \cap V \\
U \cup V\end{array}$ & $n m$ & $n m$ & $\begin{array}{ll}n m & |\Sigma| \geqslant 2 \\
\max \{n, m\} & |\Sigma|=1\end{array}$ & $\begin{array}{ll}n m & |\Sigma| \geqslant 2 \\
\max \{n, m\} & |\Sigma|=1\end{array}$ & Thm $17 \&$ [2] \\
\hline
\end{tabular}

Table 2. The state complexity results for various operations for input languages of state complexities $n$ and $m$. The upper bound $(\leqslant)$ and the best known lower bound $(\geqslant)$ are indicated for the group and the aperiodic commutative languages. Also, $f(n, m)=$ $2^{n m-1}+2^{(m-1)(n-1)}\left(2^{m-1}-1\right)\left(2^{n-1}-1\right)$ is the general bound for shuffle from [1]. So, the bound for shuffle is actually the minimum of the bound stated and $f(n, m)$ as written in Table 1 (this is left out to save horizontal space). For the lower bound for projection, consider the group language $\left(a^{n}\right)^{*} \amalg \Sigma \backslash\{a\}$ and the aperiodic language $\left\{a^{n-1}\right\} \amalg \Sigma \backslash\{a\}$ and $\Gamma \subseteq \Sigma \backslash\{a\}$.

commutative aperiodic languages separately. Additionally, we will investigate four new operations - the upward and downward closure and interior operations, denoted by $\uparrow U, \downarrow U, \Omega U$ and $\circlearrowleft U$ - for which we first state a bound on general commutative regular languages and then also bounds for commutative aperiodic and commutative group languages. See Table 1 and Table 2 for a summary of the results.

\section{Preliminaries}

In the present work, we assume that $k \geqslant 0$ denotes our alphabet size and $\Sigma=$ $\left\{a_{1}, \ldots, a_{k}\right\}$. We will also write $a, b, c$ for $a_{1}, a_{2}, a_{3}$ in case of $|\Sigma| \leqslant 3$. The set $\Sigma^{*}$ denotes the set of all finite sequences, i.e., of all words. The finite sequence of length zero, or the empty word, is denoted by $\varepsilon$. For a given word we denote by $|w|$ its length, and for $a \in \Sigma$ by $|w|_{a}$ the number of occurrences of the symbol $a$ in $w$. A language is a subset of $\Sigma^{*}$. 
The shuffle operation, denoted by $\amalg$, is defined by

$$
\begin{aligned}
& u \amalg v=\left\{w \in \Sigma^{*} \mid w=x_{1} y_{1} x_{2} y_{2} \cdots x_{n} y_{n}\right. \text { for some words } \\
& \left.\quad x_{1}, \ldots, x_{n}, y_{1}, \ldots, y_{n} \in \Sigma^{*} \text { such that } u=x_{1} x_{2} \cdots x_{n} \text { and } v=y_{1} y_{2} \cdots y_{n}\right\},
\end{aligned}
$$

for $u, v \in \Sigma^{*}$ and $L_{1} ш L_{2}:=\bigcup_{x \in L_{1}, y \in L_{2}}(x ш y)$ for $L_{1}, L_{2} \subseteq \Sigma^{*}$. If $L_{1}, \ldots, L_{n} \subseteq$ $\Sigma^{*}$, we set $\bigsqcup_{i=1}^{n} L_{i}=L_{1} \amalg \ldots \amalg L_{n}$.

Let $\Gamma \subseteq \Sigma$. The projection homomorphism $\pi_{\Gamma}: \Sigma^{*} \rightarrow \Gamma^{*}$ is the homomorphism given by $\pi_{\Gamma}(x)=x$ for $x \in \Gamma, \pi_{\Gamma}(x)=\varepsilon$ otherwise and extended by $\pi_{\Gamma}(\varepsilon)=\varepsilon$ and $\pi_{\Gamma}(w x)=\pi_{\Gamma}(w) \pi_{\Gamma}(x)$ for $w \in \Sigma^{*}$ and $x \in \Sigma$. As a shorthand, we set, with respect to a given naming $\Sigma=\left\{a_{1}, \ldots, a_{k}\right\}, \pi_{j}=\pi_{\left\{a_{j}\right\}}$. Then $\pi_{j}(w)=a_{j}^{|w|_{a_{j}}}$. For $L \subseteq \Sigma^{*}$, we set $\pi_{\Gamma}(L)=\left\{\pi_{\Gamma}(u) \mid u \in L\right\}$.

A quintuple $\mathcal{A}=\left(\Sigma, Q, \delta, q_{0}, F\right)$ is a finite deterministic and complete automaton, where $\Sigma$ is the input alphabet, $Q$ the finite set of states, $q_{0} \in Q$ the start state, $F \subseteq Q$ the set of final states and $\delta: Q \times \Sigma \rightarrow Q$ is the totally defined state transition function. The transition function $\delta: Q \times \Sigma \rightarrow Q$ extends to a transition function on words $\delta^{*}: Q \times \Sigma^{*} \rightarrow Q$ by setting $\delta^{*}(q, \varepsilon):=q$ and $\delta^{*}(q, w a):=\delta\left(\delta^{*}(q, w), a\right)$ for $q \in Q, a \in \Sigma$ and $w \in \Sigma^{*}$. In the remainder, we drop the distinction between both functions and will also denote this extension by $\delta$. Here, we do not consider incomplete automata. The language recognized by an automaton $\mathcal{A}=\left(\Sigma, Q, \delta, q_{0}, F\right)$ is $L(\mathcal{A})=\left\{w \in \Sigma^{*} \mid \delta\left(q_{0}, w\right) \in F\right\}$. A language $L \subseteq \Sigma^{*}$ is called regular if $L=L(\mathcal{A})$ for some finite automaton $\mathcal{A}$.

A language $L \subseteq \Sigma^{*}$ is a group language, if there exists a permutation automaton $\mathcal{A}=\left(\Sigma, Q, \delta, q_{0}, F\right)$, i.e., an automaton such that the map $q \mapsto \delta(q, a)$ is a permutation for each $a \in \Sigma$, recognizing $L$. A language $L \subseteq \Sigma^{*}$ is an aperiodic language, if there exists an automaton $\mathcal{A}=\left(\Sigma, Q, \delta, q_{0}, F\right)$ recognizing it such that, for each $w \in \Sigma^{*}, q \in Q$ and $n \geqslant 1$, if $\delta\left(q, w^{n}\right)=q$, then $\delta(q, w)=q$.

The Nerode right-congruence with respect to $L \subseteq \Sigma^{*}$ is defined, for $u, v \in \Sigma^{*}$, by $u \equiv_{L} v$ if and only if $\forall x \in \Sigma^{*}: u x \in L \leftrightarrow v x \in L$. The equivalence class of $w \in \Sigma^{*}$ is denoted by $[w]_{\equiv_{L}}=\left\{x \in \Sigma^{*} \mid x \equiv_{L} w\right\}$. A language is regular if and only if the above right-congruence has finite index, and it can be used to define the minimal deterministic automaton $\mathcal{A}_{L}=\left(\Sigma, Q_{L}, \delta_{L},[\varepsilon]_{\equiv_{L}}, F_{L}\right)$ with $Q_{L}=\left\{[u]_{\equiv_{L}} \mid u \in \Sigma^{*}\right\}, \delta_{L}\left([w]_{\equiv_{L}}, a\right)=[w a]_{\equiv_{L}}$ and $F_{L}=\left\{[u]_{\equiv_{L}} \mid u \in L\right\}$. Let $L \subseteq \Sigma^{*}$ be regular with minimal automaton $\mathcal{A}_{L}=\left(\Sigma, Q_{L}, \delta_{L},[\varepsilon]_{\equiv_{L}}, F_{L}\right)$. The number $\left|Q_{L}\right|$ is called the state complexity of $L$ Two words are said to be distinguishable, if they denote different right-congruence classes for a given language.

A language $L \subseteq \Sigma^{*}$ is commutative, if, for $u, v \in \Sigma^{*}$ such that $|v|_{x}=|u|_{x}$ for every $x \in \Sigma$, we have $u \in L$ if and only if $v \in L$. For commutative regular languages we have the following normal form.

Theorem 2 ([14 15]). Let $\Sigma=\left\{a_{1}, \ldots, a_{k}\right\}$ be our alphabet. A commutative language $L \subseteq \Sigma^{*}$ is regular if and only if it can be written in the form $L=$ $\bigcup_{i=1}^{n} U_{1}^{(i)} \amalg \ldots ш U_{k}^{(i)}$ with non-empty unary regular languages $U_{j}^{(i)} \subseteq\left\{a_{j}\right\}^{*}$ for $i \in\{1, \ldots, n\}$ and $j \in\{1, \ldots k\}$ that can be recognized by unary automata with a single final state. 
Let $L \subseteq \Sigma^{*}$ be a commutative regular language. For each $j \in\{1, \ldots, k\}$ let $i_{j} \geqslant 0$ and $p_{j} \geqslant 1$ be the smallest numbers such that $\left[a_{j}^{i_{j}}\right]_{\equiv_{L}}=\left[a_{j}^{i_{j}+p_{j}}\right]_{\equiv_{L}}$. The vectors $\left(i_{1}, \ldots, i_{k}\right)$ and $\left(p_{1}, \ldots, p_{k}\right)$ are then called the index and period vectors of $L$. These notions where introduced in [8]14]15] and it was shown that they can be used to bound the state complexity of $L$.

Theorem 3 ([8 14, 15]). Let $L \subseteq \Sigma^{*}$ be a commutative regular language with index vector $\left(i_{1}, \ldots, i_{k}\right)$ and period vector $\left(p_{1}, \ldots, p_{k}\right)$. Then, for any $j \in\{1, \ldots, k\}$, we have $i_{j}+p_{j} \leqslant \operatorname{sc}(L) \leqslant \prod_{r=1}^{k}\left(i_{r}+p_{r}\right)$.

Example 1. Let $L=(a a)^{*} \amalg(b b)^{*} \cup(a a a a)^{*} \amalg b^{*}$. Then $\left(i_{1}, i_{2}\right)=(0,0),\left(p_{1}, p_{2}\right)=$ $(4,2), \pi_{1}(L)=(a a)^{*}$ and $\pi_{2}(L)=b^{*}$.

The following result from 1415 connects the index and period vector with the aperiodic and group languages.

Theorem 4. A commutative regular language is:

1. aperiodic iff its period vector equals $(1, \ldots, 1)$;

2. a group language iff its index vector equals $(0, \ldots, 0)$.

Let $u, v \in \Sigma^{*}$. Then, $u$ is a subsequence 2 of $v$, denoted by $u \leqslant v$, if and only if $v \in u \amalg \Sigma^{*}$. The thereby given order is called the subsequence order. Let $L \subseteq \Sigma^{*}$. Then, we define:

1. the upward closure, by $\uparrow L=L \amalg \Sigma^{*}=\left\{u \in \Sigma^{*}: \exists v \in L: v \leqslant u\right\}$;

2. the downward closure, by $\downarrow L=\left\{u \in \Sigma^{*}: u \amalg \Sigma^{*} \cap L \neq \varnothing\right\}=\left\{u \in \Sigma^{*}\right.$ : $\exists v \in L: u \leqslant v\}$

3. the upward interior, denoted by $\circlearrowleft L$, as the largest upward-closed set in $L$, i.e. the largest subset $U \subseteq L$ such that $\uparrow U=U$;

4. the downward interior, denoted by $\Omega L$, as the largest downward-closed set in $L$, i.e., the largest subset $U \subseteq L$ such that $\downarrow U=U$.

The following equations are valid [19]:

$$
\Omega L=\Sigma^{*} \backslash \uparrow\left(\Sigma^{*} \backslash L\right) \quad \circlearrowleft L=\Sigma^{*} \backslash \downarrow\left(\Sigma^{*} \backslash L\right) .
$$

A remarkable fact is that for every language, the above closure operators give regular languages. This is based on the fact that the subsequence order is a well-order, i.e., any upward-closed set is generated by a finite subset of words 1113 .

For general $L \subseteq \Sigma^{*}$, there is no way to compute a recognizing automaton for the upward and downward operations. This is seen by an easy argument, due to 9 . Let $L \subseteq \Sigma^{*}$ be a recursively enumerable language. Then, $L$ is non-empty if and only if $\uparrow L$ is non-empty. However, the former problem is undecidable for recursively enumerable languages, but decidable for regular languages. Hence, if we can compute a recognizing automaton for $\uparrow L$, we can decide non-emptiness

\footnotetext{
${ }^{2}$ Also called a scattered subword in the literature 919.
} 
for $L$, which is, in general, not possible. But for regular and context-free $L$, recognizing automata for these operations are computable [920].

In [12, a lower bound for the state complexity of the upward closure was established by using the language $L=\bigcup_{a \in \Sigma}\left\{a^{N}\right\}$, which is commutative and finite. As every finite language is aperiodic and we are interested in this class, let us highlight this fact with the next statement.

Proposition 5 (Héam [12]). Set

$$
g(n)=\max \{\operatorname{sc}(\uparrow L) \mid \operatorname{sc}(L) \leqslant n \text { and } L \text { is finite and commutative }\} .
$$

Then $g(n) \in \Omega\left(\left(\frac{n}{|\Sigma|}\right)^{|\Sigma|}\right)$.

The next is from [14]15].

Theorem 6. Let $U, V \subseteq \Sigma^{*}$ be aperiodic commutative languages with index vectors $\left(i_{1}, \ldots, i_{k}\right)$ and $\left(j_{1}, \ldots, j_{k}\right)$. Then, $U \amalg V$ has index vector componentwise less than $\left(i_{1}+j_{1}, \ldots, i_{k}+j_{k}\right)$ and period vector $(1, \ldots, 1)$. So, $\operatorname{sc}(U \sqcup V) \leqslant$ $\prod_{l=1}^{k}\left(i_{l}+j_{l}+1\right)$. Hence, Theorem 3 yields $\operatorname{sc}(U W V) \leqslant(\operatorname{sc}(U)+\operatorname{sc}(V)-1)^{|\Sigma|}$.

As a corollary of Theorem [6 and Theorem 4, we also get, as the star-free and aperiodic languages coincide [29], an old result by J.F.Perrot [6]25]27].

Corollary 7 (J.F.Perrot [25]). The shuffle of two commutative star-free languages is star-free.

\section{The Upward and Downward Closure Operations}

In this section, we establish state complexity bounds for the upward and downward closure and interior operations. The constructions also yield polynomial time algorithms for computing those closures, if the alphabet is fixed and not allowed to vary with the input.

Theorem 8, Let $\Sigma=\left\{a_{1}, \ldots, a_{k}\right\}$. Suppose $L \subseteq \Sigma^{*}$ is commutative and regular with index vector $\left(i_{1}, \ldots, i_{k}\right)$ and period vector $\left(p_{1}, \ldots, p_{k}\right)$. Then,

$$
\max \{\operatorname{sc}(\uparrow L), \operatorname{sc}(\downarrow L), \operatorname{sc}(\circlearrowleft L), \operatorname{sc}(\bigcap L)\} \leqslant \prod_{j=1}^{k}\left(i_{j}+p_{j}\right) .
$$

Proof (sketch). We only give a rough outline of the proof idea for the first operation. For $L$, as shown in 8[14]15], we can construct an automaton of size $\prod_{j=1}^{k}\left(i_{j}+p_{j}\right)$ for $L$ whose states can be put in correspondence with the modulo and threshold counting for the different letters. More formally, it could be shown that there exists an automaton $\mathcal{C}=\left(\Sigma, S_{1}, \times \ldots \times S_{k}, \delta, s_{0}, F\right)$ with $S_{j}=\left\{0, \ldots, i_{j}+p_{j}-1\right\}$ for $j \in\{1, \ldots, k\}$ recognizing $L$. 
Construct automaton for $\uparrow L$ : Set $\mathcal{A}_{\uparrow}=\left(\Sigma, S_{1} \times \ldots \times S_{k}, \delta_{\uparrow}, s_{0}, F_{\uparrow}\right)$ with

$$
\begin{gathered}
F_{\uparrow}=\left\{\left(s_{1}, \ldots, s_{k}\right) \in S_{1} \times \ldots \times S_{k} \mid\right. \\
\left.\exists\left(f_{1}, \ldots, f_{k}\right) \in F \forall j \in\{1, \ldots, k\}: f_{j} \leqslant s_{j}\right\} ; \\
\delta_{\uparrow}\left(\left(s_{1}, \ldots, s_{k}\right), a_{j}\right)= \begin{cases}\left(s_{1}, \ldots, s_{k}\right) & \text { if } i_{j}+p_{j}-1=s_{j} ; \\
\delta\left(\left(s_{1}, \ldots, s_{k}\right), a_{j}\right) & \text { otherwise, }\end{cases}
\end{gathered}
$$

for $j \in\{1, \ldots, k\}$ and $\left(s_{1}, \ldots, s_{k}\right) \in S_{1} \times \ldots \times S_{k}$. With a similar idea, an automaton $\mathcal{A}_{\downarrow}$ such that $L\left(\mathcal{A}_{\downarrow}\right)=\downarrow L$ can be constructed. See Example 2 for concrete constructions.

Example 2. Let $L=b b(b b)^{*} \cup\left(b \amalg a(a a)^{*}\right)$. Then, $\uparrow L=b b b^{*} \cup\left(b b^{*} \amalg a a^{*}\right)$, $\downarrow L=b^{*} \cup\left(a a^{*} \amalg\{\varepsilon, b\}\right), \circlearrowleft L=\varnothing$ and $\Omega L=\varnothing$. The constructions of the automata $\mathcal{A}_{\uparrow}$ and $\mathcal{A}_{\downarrow}$ with $L\left(\mathcal{A}_{\uparrow}\right)=\uparrow L$ and $L\left(\mathcal{A}_{\downarrow}\right)=\downarrow L$ from the proof sketch of Theorem 8 are illustrated, for the example language $L$, in Figure 1 .
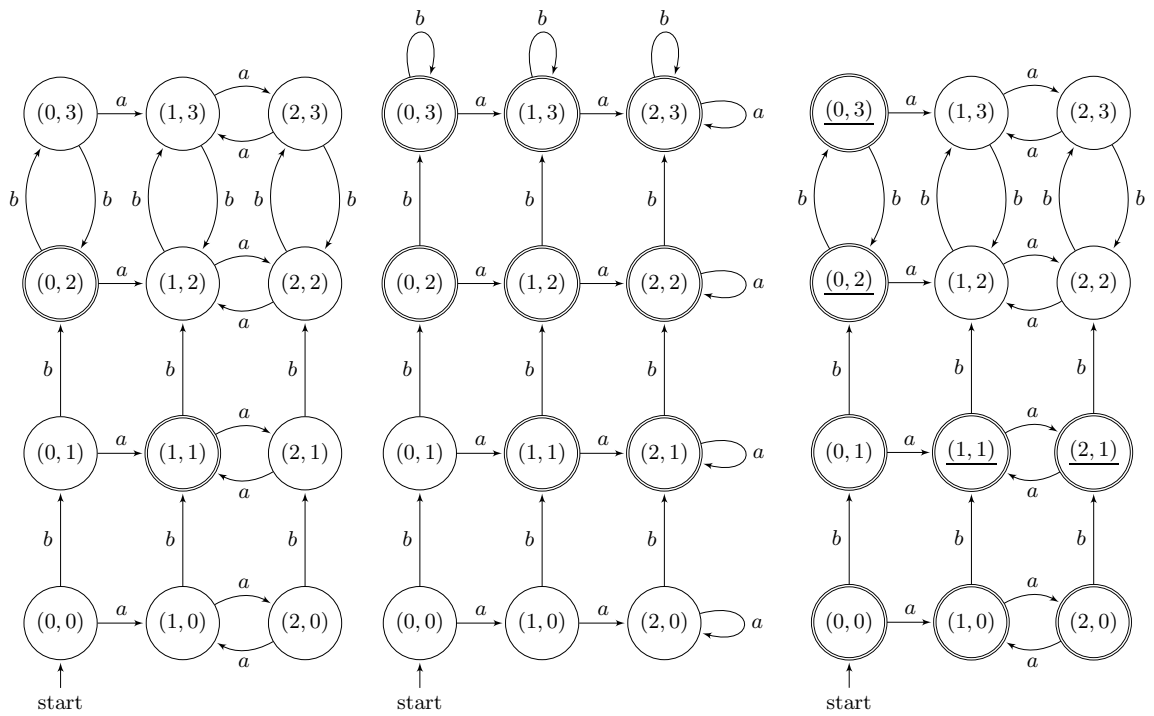

Fig. 1. Construction of automata for the upward and downward closure for the language $L=b b(b b)^{*} \cup\left(b \amalg a(a a)^{*}\right)$ by starting from an automaton in a "rectangular" normal form for $L$. The left-most automaton recognizes $L$, the automaton in the middle recognized $\uparrow L$ and the right-most automaton recognized $\downarrow L$. See Example 2 for details.

The constructions done in the proof of Theorem 8 can actually be performed in polynomial time.

Corollary 9. Fix the alphabet $\Sigma$. Let $L \subseteq \Sigma^{*}$ be commutative and regular, given by a finite recognizing automaton with $n$ states. Then, recognizing automata for $\uparrow L, \downarrow L, \Omega L$ and $\circlearrowleft L$ are computable in polynomial time in $n$. 
With Theorem 3 , we can derive the next bound from Theorem 8 .

Theorem 10. Let $L \subseteq \Sigma^{*}$ be commutative and recognizable by an automaton with $n$ states. Then, the upward and downward closures and interiors of $L$ are recognizable by automata of size $n^{|\Sigma|}$.

\section{The Case of Commutative Group Languages}

Before we investigate the state complexity of shuffle, union, intersection and the closure and interior operations for commutative group languages, we give a normal form theorem for commutative groups languages similar to Theorem 2 ,

Theorem 11, Let $\Sigma=\left\{a_{1}, \ldots, a_{k}\right\}$ and $L \subseteq \Sigma^{*}$. Then, the following conditions are equivalent:

1. L is a commutative group language;

2. $L$ is a finite union of languages of the form $U_{1} \amalg \ldots \amalg U_{k}$ with $U_{j} \subseteq\left\{a_{j}\right\}^{*}$ a group language recognizable by an automaton with a single final state;

3. $L$ is a finite union of languages of the form $U_{1} \amalg \ldots \amalg U_{k}$ with $U_{j} \subseteq\left\{a_{j}\right\}^{*}$ being group languages.

\subsection{The Shuffle Operation}

Here, we give a sharp bound for the state complexity of two commutative group languages. However, in this case, we do not express the bound in terms of the size of recognizing input automata, but in terms of the index and period vectors of the input languages. The result generalizes a corresponding result from $[26]$ for unary group languages to commutative group languages.

Theorem [12, Let $\Sigma=\left\{a_{1}, \ldots, a_{k}\right\}$. For commutative group languages $U, V \subseteq$ $\Sigma^{*}$ with period vectors $\left(p_{1}, \ldots, p_{k}\right)$ and $\left(q_{1}, \ldots, q_{k}\right)$ their shuffle $U \omega V$ has index vector $\left(i_{1}, \ldots, i_{k}\right)$ with $i_{j}=\operatorname{lcm}\left(p_{j}, q_{j}\right)-1$ for $j \in\{1, \ldots, k\}$ and period vector $\left(\operatorname{gcd}\left(p_{1}, q_{1}\right), \ldots, \operatorname{gcd}\left(p_{k}, q_{k}\right)\right)$. Hence, by Theorem $\mathbf{3}$,

$$
\operatorname{sc}(U ш V) \leqslant \prod_{j=1}^{k}\left(\operatorname{gcd}\left(p_{j}, q_{j}\right)+\operatorname{lcm}\left(p_{j}, q_{j}\right)-1\right) .
$$

Furthermore, there exist commutative group languages such that a minimal automaton recognizing their shuffle reaches the bound.

As for any two numbers $n, m>0$ we always have $\operatorname{gcd}(n, m)+\operatorname{lcm}(n, m)-1 \leqslant$ $\mathrm{nm}$, we can deduce the next bound in terms of the size of recognizing automata. The result improves the general bound $(2 \mathrm{~nm})^{|\Sigma|}$ from 1415 .

Theorem 13. Let $U, V \subseteq \Sigma^{*}$ be commutative group languages recognized by automata with $n$ and $m$ states. Then, $U \amalg V$ is recognizable by an automaton with at most $(\mathrm{nm})^{|\Sigma|}$ states. 
We do not know if the last bound is sharp. The best lower bound we can give is the next one, which essentially follows by the lower bound for concatenation in case of unary languages, see [31, Theorem 5.4].

Proposition 14. Let $n, m>0$ be coprime numbers. Then, there exist commutative group languages of states complexities $n$ and $m$ such that their shuffle has state complexity $\mathrm{nm}$.

\subsection{The Upward and Downward Closure and Interior Operations}

First, we will show that every word is contained in the downward closure of a commutative group language.

Proposition 15. Let $L \subseteq \Sigma^{*}$ be a commutative group language. Then the downward closure $\downarrow L$ equals $\Sigma^{*}$.

This is not true for general commutative languages, see Proposition 19,

Proposition 16. Let $n>0$. There exists a commutative group language $L \subseteq$ $\Sigma^{*}$ with period vector $(n, 1, \ldots, 1)$ such that $\operatorname{sc}(L)=n$ and its upward closure has state complexity $n$ with index vector $(n-1,1, \ldots, 1)$ and period vector $(1, \ldots, 1)$.

Proof. Let $a \in \Sigma$ and $n>0$. Set $L=\left\{\left.w \in \Sigma^{*}|| w\right|_{a} \equiv n-1(\bmod n)\right\}=$ $a^{n-1}\left(a^{n}\right)^{*} \amalg(\Sigma \backslash\{a\})^{*}$. Then, $\uparrow L=a^{n-1} a^{*} \amalg(\Sigma \backslash\{a\})^{*}$ and $\operatorname{sc}(\uparrow L)=n$.

\subsection{Union and Intersection}

Theorem 17. For any alphabet $\Sigma$ and commutative group language of state complexities $n$ and $m$, the intersection and union is recognizable by an automaton with $\mathrm{nm}$ states. Furthermore, there exists commutative group languages with state complexities such that every automaton for their union (intersection) needs nm states.

Proof. The upper bound holds for regular languages in general [31. Let $a \in \Sigma$ and $n, m>0$ two coprime numbers. The languages for the lower bound are similar to the ones given in 31, but also work for $\Sigma=\{a\}$ (in 31] $|\Sigma| \geqslant 2$ is assumed). Set $U=\left\{\left.w \in \Sigma^{*}|| w\right|_{a} \equiv 0(\bmod n)\right\}=\left(a^{n}\right)^{*} w(\Sigma \backslash\{a\})^{*}$ and $V=\left\{\left.w \in \Sigma^{*}|| w\right|_{a} \equiv 0(\bmod m)\right\}$. Then, $\operatorname{sc}(U \cap V)=\operatorname{sc}(U \cup V)=n m$.

\section{The Case of Commutative Aperiodic Languages}

For shuffle (Theorem 6), union and intersection (see [31, Theorem 4.3]), we already have upper bounds, see Table 2. Note that the lower bound construction for Boolean operations given in [2, Theorem $1 \& 8$ ] over an at least binary alphabet uses the commutative and aperiodic languages $\left\{\left.w \in \Sigma^{*}|| w\right|_{a}=n-2\right\}$ and $\left\{\left.w \in \Sigma^{*}|| w\right|_{b}=m-2\right\}$ for $n, m \geqslant 2$. For the upward closure, Proposition 5 
gives a lower bound. So, here, we handle the missing cases of the downward closure and the shuffle operation.

But first, we give a similar normal form theorem for aperiodic commutative languages as for group languages. The proof is essentially the same as in the group case.

Theorem 18. Let $\Sigma=\left\{a_{1}, \ldots, a_{k}\right\}$ and $L \subseteq \Sigma^{*}$. Then, the following conditions are equivalent:

1. L is a commutative and aperiodic language;

2. $L$ is a finite union of languages of the form $U_{1} \amalg \ldots \amalg U_{k}$ with $U_{j} \subseteq\left\{a_{j}\right\}^{*}$ aperiodic and recognizable by an automaton with a single final state;

3. $L$ is a finite union of languages of the form $U_{1} \amalg \ldots ш U_{k}$ with $U_{j} \subseteq\left\{a_{j}\right\}^{*}$ aperiodic.

Next, we state a lower bound for the downward closure. By Equation (11), using the complemented language, this implies the same lower bound for the upward interior.

Proposition 19. Let $a \in \Sigma$ and $n>0$. Set $L=\left\{a^{n}\right\}$. Then, $\downarrow L=\left\{\varepsilon, a, \ldots, a^{n}\right\}$ and so $\operatorname{sc}(\downarrow L)=\operatorname{sc}(L)=n+2$.

For concatenation it is known that the state complexity for unary languages is $n m$ [31, Theorem 5.4 \& Theorem 5.5]. In fact, the witness languages for the lower bound are group languages (see also Prop 14). For unary aperiodic languages, concatenation has state complexity $n+m-1$ [2, Theorem 8]. By using these unary witness languages and introducing self-loops for additional letters, and as in the unary case (and for the mentioned extension to more letters) concatenation and shuffle coincide, this immediately gives lower bounds for the shuffle operation on commutative languages as well.

However, in the next result, we show that we can do better for aperiodic (even for finite) commutative languages.

Proposition 20. Let $\Sigma$ be an at least binary alphabet. Then for each even $n, m>0$ there exist commutative and finite languages $U, V \subseteq \Sigma^{*}$ with $\operatorname{sc}(U)=$ $n, \operatorname{sc}(V)=m$ such that $\operatorname{sc}(U \amalg V) \geqslant \frac{n m}{4}+1$.

Proof. We give the construction for a binary alphabet, as for larger alphabets the lower bound is implied by adding self-loops to the automaton for each additional letter. Let $\Sigma=\{a, b\}$ and $N, M \geqslant 0$ with $n=|\Sigma| N+2$ and $m=|\Sigma| M+2$. Set $U=\left\{a^{N}, b^{N}\right\}$ and $V=\left\{a^{M}, b^{M}\right\}$. Then $\operatorname{sc}(U)=n$ and $\operatorname{sc}(V)=m$. Set

$$
\begin{aligned}
L=U ш V=\left\{u \in \Sigma^{*} \mid\right. & \left(|u|_{a}=N+M,|u|_{b}=0\right) \vee\left(|u|_{a}=N,|u|_{b}=M\right) \\
& \left.\vee\left(|u|_{a}=M,|u|_{b}=N\right) \vee\left(|u|_{a}=0,|u|_{b}=N+M\right)\right\} .
\end{aligned}
$$

We show that the words $a^{i} b^{j}$ for $i \in\{0,1, \ldots, N\}$ and $j \in\{0,1, \ldots, M\}$ are pairwise inequivalent for the Nerode right-congruence. Let $\left(n_{1}, n_{2}\right),\left(m_{1}, m_{2}\right) \in$ $\{0,1, \ldots, N\} \times\{0,1, \ldots, M\}$ with $\left(n_{1}, n_{2}\right) \neq\left(m_{1}, m_{2}\right)$. First, suppose $n_{i} \neq 0$ for 
$i \in\{0,1\}$ and $m_{i} \neq 0$ for $i \in\{0,1\}$. Set $u=a^{N-n_{1}} b^{M-n_{2}}$. Then $a^{n_{1}} b^{n_{2}} u \in L$. By the assumptions, $a^{m_{1}} b^{m_{2}} u \in L$ if and only if $\left(m_{1}, m_{2}\right)+\left(N-n_{1}, M-n_{2}\right)=$ $(M, N)$. So, if this is not the case, then $u$ distinguishes both words. Otherwise, we must have $\left(n_{1}, n_{2}\right)+\left(N-m_{1}, M-m_{2}\right) \neq(M, N)$, for if not then $M-N=$ $n_{1}-m_{1}=m_{1}-n_{1}$. The last equality then gives $n_{1}=m_{1}$ and similarly we find $n_{2}=m_{2}$. So, in that case with $v=a^{N-m_{1}} b^{M-m_{2}}$ we have $a^{m_{1}} b^{m_{2}} v \in L$ but $a^{n_{1}} b^{n_{2}} v \notin L$. Lastly, if at least one component is zero, we must distinguish more cases as $\left\{a^{N+M}, b^{N+M}\right\} \subseteq L$, which are left out here due to space.

So, we have $\operatorname{sc}(L) \geqslant(N+1)(M+1)+1$ (the additional one accounts for a trap state necessary, as we measure the state complexity in terms of complete automata). Hence, $\operatorname{sc}(L) \geqslant \frac{n m}{4}+1$.

\section{Conclusion}

As shown in Table 1 and Table 2, for shuffle and the upward and downward closure and interior operations, it is not known if the given upper bounds are tight. Hence, this is an open problem.

Acknowledgement. I thank the anonymous referees of [14] (the extended version of [15]) whose feedback also helped in the present work. I also thank the referees of the present work for critical and careful reading, and pointing out typos and parts that needed better explanation.

\section{References}

1. Brzozowski, J.A., Jirásková, G., Liu, B., Rajasekaran, A., Szykuła, M.: On the state complexity of the shuffle of regular languages. In: Câmpeanu, C., Manea, F., Shallit, J.O. (eds.) DCFS 2016. LNCS, vol. 9777, pp. 73-86. Springer (2016)

2. Brzozowski, J.A., Liu, B.: Quotient complexity of star-free languages. International Journal of Foundations of Computer Science 23(6), 1261-1276 (2012)

3. Brzozowski, J.A., Simon, I.: Characterizations of locally testable events. Discrete Mathematics 4(3), 243-271 (1973)

4. Campbell, R.H., Habermann, A.N.: The specification of process synchronization by path expressions. In: Gelenbe, E., Kaiser, C. (eds.) Operating Systems OS. LNCS, vol. 16, pp. 89-102. Springer (1974)

5. Câmpeanu, C., Salomaa, K., Yu, S.: Tight lower bound for the state complexity of shuffle of regular languages. J. Autom. Lang. Comb. 7(3), 303-310 (2002)

6. Castiglione, G., Restivo, A.: On the shuffle of star-free languages. Fundam. Informaticae 116(1-4), 35-44 (2012)

7. Gao, Y., Moreira, N., Reis, R., Yu, S.: A survey on operational state complexity. Journal of Automata, Languages and Combinatorics 21(4), 251-310 (2017)

8. Gómez, A.C., Alvarez, G.I.: Learning commutative regular languages. In: Clark, A., Coste, F., Miclet, L. (eds.) ICGI 2008. LNCS, vol. 5278, pp. 71-83. Springer (2008)

9. Gruber, H., Holzer, M., Kutrib, M.: The size of Higman-Haines sets. Theor. Comput. Sci. 387(2), 167-176 (2007)

10. Gruber, H., Holzer, M., Kutrib, M.: More on the size of Higman-Haines sets: Effective constructions. Fundam. Informaticae 91(1), 105-121 (2009) 
11. Haines, L.H.: On free monoids partially ordered by embedding. Journal of Combinatorial Theory 6, 94-98 (1969)

12. Héam, P.: On shuffle ideals. RAIRO Theor. Informatics Appl. 36(4), 359-384 (2002)

13. Higman, G.: Ordering by divisibility in abstract algebras. Proceedings of the London Mathematical Society (3) 2(7), 326-336 (1952)

14. Hoffmann, S.: Commutative regular languages - properties and state complexity. Information and Computation (to appear)

15. Hoffmann, S.: Commutative regular languages - properties and state complexity. In: Ciric, M., Droste, M., Pin, J. (eds.) CAI 2019, Niš, Serbia, June 30 - July 4, 2019, Proceedings. LNCS, vol. 11545, pp. 151-163. Springer (2019)

16. Hoffmann, S.: Commutative regular languages with product-form minimal automata. In: Han, Y.S., Ko, S.K. (eds.) DCFS 2021, Proceedings. LNCS, Springer (2021)

17. Hoffmann, S.: State complexity of projection on languages recognized by permutation automata and commuting letters. In: DLT 2021, Porto, Portugal, August, 16-20, 2021, Proceedings (2021)

18. Hospodár, M., Mlynárčik, P.: Operations on permutation automata. In: Jonoska, N., Savchuk, D. (eds.) DLT 2020, Tampa, FL, USA, May 11-15, 2020, Proceedings. Lecture Notes in Computer Science, vol. 12086, pp. 122-136. Springer (2020)

19. Karandikar, P., Niewerth, M., Schnoebelen, P.: On the state complexity of closures and interiors of regular languages with subwords and superwords. Theoretical Computer Science 610, 91-107 (2016)

20. van Leeuwen, J.: Effective constructions in well-partially- ordered free monoids. Discret. Math. 21(3), 237-252 (1978)

21. Maslov, A.N.: Estimates of the number of states of finite automata. Dokl. Akad. Nauk SSSR 194(6), 1266-1268 (1970)

22. Mazurkiewicz, A.W.: Parallel recursive program schemes. In: Becvár, J. (ed.) MFCS 1975, Proceedings. LNCS, vol. 32, pp. 75-87. Springer (1975)

23. McNaughton, R.: The loop complexity of pure-group events. Information and Control 11(1/2), 167-176 (1967)

24. Okhotin, A.: On the state complexity of scattered substrings and superstrings. Fundam. Informaticae 99(3), 325-338 (2010)

25. Perrot, J.: Varietes de langages et operations. Theor. Comput. Sci. 7, 197-210 (1978)

26. Pighizzini, G., Shallit, J.: Unary language operations, state complexity and jacobsthal's function. International Journal of Foundations of Computer Science 13(1), 145-159 (2002)

27. Restivo, A.: The shuffle product: New research directions. In: Dediu, A., Formenti, E., Martín-Vide, C., Truthe, B. (eds.) LATA 2015. LNCS, vol. 8977, pp. 70-81. Springer (2015)

28. Riddle, W.E.: An approach to software system behavior description. Comput. Lang. 4(1), 29-47 (1979)

29. Schützenberger, M.P.: On finite monoids having only trivial subgroups. Inf. Control. 8(2), 190-194 (1965)

30. Shaw, A.C.: Software descriptions with flow expressions. IEEE Trans. Softw. Eng. 4, 242-254 (1978)

31. Yu, S., Zhuang, Q., Salomaa, K.: The state complexities of some basic operations on regular languages. Theoretical Computer Science 125(2), 315-328 (Mar 1994) 


\section{A Proofs for Section 2 (Preliminaries)}

An automaton $\mathcal{A}=\left(\Sigma, Q, \delta, q_{0}, F\right)$ is called commutative, if, for each $q \in Q$ and $a, b \in \Sigma$, we have $\delta(q, a b)=\delta(q, b a)$.

\section{B Proofs for Section 3 (The Upward and Downward Closure Operations)}

The Parikh map is the map $\psi: \Sigma^{*} \rightarrow \mathbb{N}_{0}^{k}$ given by $\psi(w)=\left(|w|_{a_{1}}, \ldots,|w|_{a_{k}}\right)$ for $w \in \Sigma^{*}$. If $L$ is commutative, then $L$ can be identified with its Parikh image $\psi(L)$ and we have

$$
u \in \downarrow L \Leftrightarrow \exists v \in L: \psi(u) \leqslant \psi(v) \text { and } u \in \uparrow L \Leftrightarrow \exists v \in L: \psi(v) \leqslant \psi(u) .
$$

For $L \subseteq \Sigma^{*}$, we set perm $(L)=\psi^{-1}(\psi(L))$, the commutative closure of $L$.

We need the following construction from [GA08].

Let $\Sigma=\left\{a_{1}, \ldots, a_{k}\right\}$ be our finite alphabet 3 . The minimal commutative automator 4 for a commutative language was introduced in [GA08].

Definition 21 (minimal commutative automaton). Let $L \subseteq \Sigma^{*}$. The minimal commutative automaton is $\mathcal{C}_{L}=\left(\Sigma, S_{1} \times \ldots \times S_{k}, \delta, s_{0}, F\right)$ with

$$
S_{j}=\left\{\left[a_{j}^{m}\right]_{\equiv_{L}}: m \geqslant 0\right\}, \quad F=\left\{\left(\left[\pi_{1}(w)\right]_{\equiv_{L}}, \ldots,\left[\pi_{k}(w)\right]_{\equiv_{L}}\right): w \in L\right\}
$$

and $\delta\left(\left(s_{1}, \ldots, s_{j}, \ldots, s_{k}\right), a_{j}\right)=\left(s_{1}, \ldots, \delta_{j}\left(s_{j}, a_{j}\right), \ldots, s_{k}\right)$ with one-letter transitions $\delta_{j}\left(\left[a_{j}^{m}\right]_{\equiv_{L}}, a_{j}\right)=\left[a_{j}^{m+1}\right]_{\equiv_{L}}$ for $j=1, \ldots, k$ and $s_{0}=\left([\varepsilon]_{\equiv_{L}}, \ldots,[\varepsilon]_{\equiv_{L}}\right)$.

Remark 1. Let $L \subseteq \Sigma^{*}$ be commutative and $\mathcal{C}_{L}=\left(\Sigma, S_{1} \times \ldots \times S_{k}, \delta, s_{0}, F\right)$ be the minimal commutative automaton. Note that, by the definition of the transition function in Definition 21, we have, for any $u, v \in \Sigma^{*}$,

$$
\delta\left(s_{0}, u\right)=\delta\left(s_{0}, v\right) \Leftrightarrow \forall j \in\{1, \ldots, k\}: \pi_{j}(u) \equiv_{L} \pi_{j}(v) .
$$

If $L$ is commutative, in [GA08] it was shown that this notion is well-defined and recognizes $L$, and it was noted that in general the minimal commutative automaton is not equal to the minimal deterministic and complete automaton for $L$.

Theorem 22 (Gómez \& Alvarez [GA08]). Let $L \subseteq \Sigma^{*}$ be a commutative language. Then, $L=L\left(\mathcal{C}_{L}\right)$ and $L$ is regular if and only if $\mathcal{C}_{L}$ is finite.

\footnotetext{
${ }^{3}$ Recall that we might write $\Sigma=\{a, b\}$ as a shorthand for $\Sigma=\left\{a_{1}, a_{2}\right\}$ in examples.

${ }^{4}$ The minimal commutative automaton is a commutative automaton in the sense of [BS73]. However, also the minimal automaton of a commutative language is commutative in this sense [FH19]. So, naming the construction to come the minimal commutative automaton might be a bit misleading, probably minimal canonical commutative automaton being a better choice. Nevertheless, I will stick to the terminology as introduced in [GA08].
} 
Theorem 8. Let $\Sigma=\left\{a_{1}, \ldots, a_{k}\right\}$. Suppose $L \subseteq \Sigma^{*}$ is commutative and regular with index vector $\left(i_{1}, \ldots, i_{k}\right)$ and period vector $\left(p_{1}, \ldots, p_{k}\right)$. Then,

$$
\max \{\operatorname{sc}(\uparrow L), \operatorname{sc}(\downarrow L), \operatorname{sc}(\circlearrowleft L), \operatorname{sc}(\Omega L)\} \leqslant \prod_{j=1}^{k}\left(i_{j}+p_{j}\right) .
$$

Proof. Let $\mathcal{C}_{L}=\left(\Sigma, S_{1}, \times \ldots \times S_{k}, \delta, s_{0}, F\right)$ be the minimal commutative automaton of $L$. For notational simplicity, we identify its states with tuples of natural numbers. More precisely, we assume $S_{j}=\left\{0, \ldots, i_{j}+p_{j}-1\right\}$ for $j \in\{1, \ldots, k\}$, where $n \in S_{j}$ corresponds to the Nerode right-congruence class $\left[a_{j}^{n}\right]_{\equiv_{L}}$. With this identification 5 , for $w \in \Sigma^{*}$ with $\psi(w) \leqslant\left(i_{1}+p_{1}-1, \ldots, i_{k}+p_{k}-1\right)$, we have

$$
\delta\left(s_{0}, w\right)=\psi(w)
$$

And for arbitrary $u \in \Sigma^{*}$,

$$
\delta\left(s_{0}, u\right) \leqslant \psi(u) .
$$

Also, if $\delta\left(s_{0}, w\right)=\left(s_{1}, \ldots, s_{k}\right)$, then, for $j \in\{1, \ldots, k\}$,

$$
\left(\left(s_{j}<i_{j}\right) \vee\left(\left|\pi_{j}(w)\right|<i_{j}+p_{j}\right)\right) \Rightarrow\left(s_{j}=\left|\pi_{j}(w)\right| \wedge \delta\left(s_{0}, \pi_{j}(w)\right)=\left|\pi_{j}(w)\right|\right) .
$$

1. Set $\mathcal{A}_{\uparrow}=\left(\Sigma, S_{1} \times \ldots \times S_{k}, \delta_{\uparrow}, s_{0}, F_{\uparrow}\right)$ with, for $j \in\{1, \ldots, k\}$ and $\left(s_{1}, \ldots, s_{k}\right) \in$ $S_{1} \times \ldots \times S_{k}$,

$$
\begin{aligned}
F_{\uparrow} & =\left\{s \in S_{1} \times \ldots \times S_{k} \mid \exists f \in F: f \leqslant s\right\} ; \\
\delta_{\uparrow}\left(\left(s_{1}, \ldots, s_{k}\right), a_{j}\right) & = \begin{cases}\left(s_{1}, \ldots, s_{k}\right) & \text { if } i_{j}+p_{j}-1=s_{j} ; \\
\delta\left(\left(s_{1}, \ldots, s_{k}\right), a_{j}\right) & \text { otherwise. }\end{cases}
\end{aligned}
$$

Then, $\mathcal{A}_{\uparrow}$ has the following properties: for any $u, v \in \Sigma^{*}$,

$$
\psi(u) \leqslant \psi(v) \Rightarrow \delta_{\uparrow}\left(s_{0}, u\right) \leqslant \delta_{\uparrow}\left(s_{0}, v\right)
$$

and, more generally, for $w \in \Sigma^{*}$,

$$
\delta_{\uparrow}\left(s_{0}, w\right)=\left(\max \left\{|w|_{a_{1}}, i_{1}+p_{1}-1\right\}, \ldots, \max \left\{|w|_{a_{k}}, i_{k}+p_{k}-1\right\}\right) .
$$

Equation (10) also implies, for any $w \in \Sigma^{*}$,

$$
\delta_{\uparrow}\left(s_{0}, w\right) \leqslant \psi(w) .
$$

Claim: We have $\uparrow L=L\left(\mathcal{A}_{\uparrow}\right)$.

Proof of the Claim: Suppose $w \in L\left(\mathcal{A}_{\uparrow}\right)$. Then, $\delta_{\uparrow}\left(s_{0}, w\right) \in F_{\uparrow}$. So, there exists $f \in F$ with $f \leqslant \delta_{\uparrow}\left(s_{0}, w\right)$. Let $u \in \Sigma^{*}$ be minimal with $\delta_{\uparrow}\left(s_{0}, u\right)=f$. Then, by Equation (10) and the minimality of $u$, we have, for any $j \in\{1, \ldots, k\}$, that $0 \leqslant|u|_{a_{j}} \leqslant i_{j}+p_{j}-1$, or $\psi(u) \leqslant\left(i_{1}+p_{1}-1, \ldots, i_{k}+p_{k}-1\right)$. So, by Equation (10), we find $f=$

\footnotetext{
${ }^{5}$ Recall that $\mathbb{N}_{0}^{k}$ is equipped with the component-wise order.
} 
$\delta_{\uparrow}\left(s_{0}, u\right)=\psi(u)$. Also, by Equation (4),$\delta\left(s_{0}, u\right)=\psi(u)=f$. Hence, $u \in L$. Then, combining the previous facts and using Equation (11),

$$
\psi(u)=f \leqslant \delta_{\uparrow}\left(s_{0}, w\right) \leqslant \psi(w) .
$$

So, by Equation (2), $w \in \uparrow L$.

Conversely, suppose $w \in \uparrow L=L \amalg \Sigma^{*}$. Then, some minimal subsequence $u$ of $w$ drives $\mathcal{C}_{L}$ into a final state, i.e., $\delta\left(s_{0}, u\right) \in F$. We have $\psi(u) \leqslant \psi(w)$. Hence, by Equation (9),

$$
\delta_{\uparrow}\left(s_{0}, u\right) \leqslant \delta_{\uparrow}\left(s_{0}, w\right) .
$$

Assume $|u|_{a_{j}}>i_{j}+p_{j}-1$ for some $j \in\{1, \ldots, k\}$. Set $m=|u|_{a_{j}}-p_{j}$. Then, $\delta\left(s_{0}, u\right)=\delta\left(s_{0}, \pi_{1}(u) \cdots \pi_{j-1}(u) a_{j}^{m} \pi_{j+1}(u) \cdots \pi_{k}(u)\right)$ and so, by commutativity, by leaving out $p_{j}$ many times the letter $a_{j}$ in $u$ we find a shorter word that ends in the same state. However, this is excluded by minimality of $u$. Hence, $\psi(u) \leqslant\left(i_{1}+p_{1}-1, \ldots, i_{k}+\right.$ $\left.p_{k}-1\right)$. So, by Equation (10), $\psi(u)=\delta_{\uparrow}\left(s_{0}, u\right)$ and, by Equation (4), $\delta\left(s_{0}, u\right)=\psi(u)$. Therefore,

$$
\delta_{\uparrow}\left(s_{0}, u\right)=\psi(u)=\delta\left(s_{0}, u\right) \in F .
$$

As $\delta_{\uparrow}\left(s_{0}, u\right) \leqslant \delta_{\uparrow}\left(s_{0}, w\right)$ and $\delta_{\uparrow}\left(s_{0}, u\right) \in F$, we have $\delta_{\uparrow}\left(s_{0}, w\right) \in F_{\uparrow}$. Hence, $w \in L\left(\mathcal{A}_{\uparrow}\right)$. [End, Proof of the Claim $]$

2. Set $\mathcal{A}_{\downarrow}=\left(\Sigma, S_{1} \times \ldots \times S_{k}, \delta, s_{0}, F_{\downarrow}\right)$ with 6

$$
F_{\downarrow}=\left\{s \in S_{1} \times \ldots \times S_{k} \mid \exists e \in E^{\prime}: s \leqslant e\right\},
$$

where

$$
\begin{aligned}
E^{\prime}=\{ & \left(s_{1}, \ldots, s_{k}\right) \in S_{1} \times \ldots \times S_{k} \\
& \exists\left(f_{1}, \ldots, f_{k}\right) \in F \forall j \in\{1, \ldots, k\}: \\
& \left.\left(\left(i_{j} \leqslant s_{j}<i_{j}+p_{j}\right) \wedge\left(i_{j} \leqslant f_{j}<i_{j}+p_{j}\right)\right) \vee\left(f_{j}=s_{j}\right)\right\} .
\end{aligned}
$$

Note that, $\left(s_{1}, \ldots, s_{k}\right) \in E^{\prime}$ if and only if there exists $\left(f_{1}, \ldots, f_{k}\right) \in F$ such that, for any $j \in\{1, \ldots, k\}$, either

$$
\left(i_{j} \leqslant s_{j}<i_{j}+p_{j}\right) \wedge\left(i_{j} \leqslant f_{j}<i_{j}+p_{j}\right)
$$

or

$$
\left(\max \left\{f_{j}, s_{j}\right\}<i_{j}\right) \wedge\left(f_{j}=s_{j}\right) .
$$

Claim: We have $\downarrow L=L\left(\mathcal{A}_{\downarrow}\right)$.

Proof of the Claim: Suppose $w \in L\left(\mathcal{A}_{\downarrow}\right)$. Set $\left(s_{1}, \ldots, s_{k}\right)=\delta\left(s_{0}, w\right)$.

We have two cases:

${ }^{6}$ Observe that we retain the transition function of $\mathcal{C}_{L}$. In $\mathcal{A}_{\downarrow}$, only the final state set is altered. 
(a) $\left(s_{1}, \ldots, s_{k}\right) \in E^{\prime}$.

We first show the auxiliary statement (i), and use this to construct a word $u \in L$ with $w \leqslant u$.

(i) We claim that there exists $\left(f_{1}, \ldots, f_{k}\right) \in F$ such that, for any $j \in\{1, \ldots, k\}$, we can find $m_{j} \geqslant 0$ with $m_{j} \geqslant\left|\pi_{j}(w)\right| \geqslant s_{j}$ and

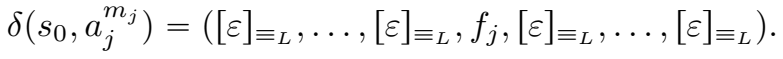

Proof of (i): Intuitively, if $\left(s_{1}, \ldots, s_{k}\right) \in E^{\prime}$, for some state from $F$, each entry $s_{j}, j \in\{1, \ldots, k\}$, either equals the $j$-th entry in the state from $F$ or shares a cycle with the $j$-th entry in the state from $F$. More formally, by the definition of $E^{\prime}$, there exists $f=\left(f_{1}, \ldots, f_{k}\right) \in F$ such that, for any $j \in\{1, \ldots, k\}$,

$$
\left(\left(i_{j} \leqslant s_{j}<i_{j}+p_{j}\right) \wedge\left(i_{j} \leqslant f_{j}<i_{j}+p_{j}\right)\right) \text { or }\left(s_{j}=f_{j}\right) .
$$

Set $r=|w|$ and define a vector $\left(m_{1}, \ldots, m_{k}\right) \in \mathbb{N}^{k}$ according to the following rules, based on the definition of $E^{\prime}$,

$m_{j}= \begin{cases}f_{j} & \text { if } f_{j}=s_{j} \\ f_{j}+r \cdot p_{j} & \text { if }\left(i_{j} \leqslant s_{j}<i_{j}+p_{j}\right) \wedge\left(i_{j} \leqslant f_{j}<i_{j}+p_{j}\right) .\end{cases}$

Then, Equation (13) holds true. By Equation (5), we have $s_{j} \leqslant\left|\pi_{j}(w)\right|$. We only need to show $m_{j} \geqslant\left|\pi_{j}(w)\right|$. Let $j \in$ $\{1, \ldots, k\}$. If $\left(i_{j} \leqslant s_{j}<i_{j}+p_{j}\right) \wedge\left(i_{j} \leqslant f_{j}<i_{j}+p_{j}\right)$, as $p_{j}>0$ and by choice of $r$ we have $m_{j} \geqslant\left|\pi_{j}(w)\right|$. Otherwise, we must have $s_{j}=f_{j}$ and, by Equation (12), $s_{j}<i_{j}$. So, by Equation (6) and the definition of $m_{j}, m_{j}=f_{j}=s_{j}=$ $\left|\pi_{j}(w)\right| .[$ End, Proof of $(i)]$

Set $u=a_{1}^{m_{1}} \cdot \ldots \cdot a_{k}^{m_{k}}$. By Definition 21 and Equation (13), $\delta\left(s_{0}, u\right)=\left(f_{1}, \ldots, f_{k}\right)$. Hence, $u \in L$, and, as $m_{j} \geqslant\left|\pi_{j}(w)\right|, j \in$ $\{1, \ldots, k\}$, we find $w ш \Sigma^{*} \cap \operatorname{perm}(u) \neq \varnothing$, and, as $\operatorname{perm}(u) \subseteq L$, $w \amalg \Sigma^{*} \cap L \neq \varnothing$. Hence, $w \in \downarrow L$.

(b) $\left(s_{1}, \ldots, s_{k}\right) \in F_{\downarrow} \backslash E^{\prime}$.

Then, we find $e=\left(e_{1}, \ldots, e_{k}\right) \in E^{\prime}$ such that $\delta\left(s_{0}, w\right) \leqslant e$. Write $e=\left(e_{1}, \ldots, e_{k}\right)$ and $\delta\left(s_{0}, w\right)=\left(s_{1}, \ldots, s_{k}\right)$. Set

$$
u=w a_{1}^{e_{1}-s_{1}} \cdot \ldots \cdot a_{k}^{e_{k}-s_{k}} .
$$

Then, $\delta\left(s_{0}, u\right) \in E^{\prime}$. By the first case, as this handled the case of words that end in a state in $E^{\prime}$, we can deduce $u \in \downarrow L$. But then, as $w \preccurlyeq u$, we also have $w \in \downarrow L$. 
Conversely, suppose $w \in \downarrow L$. Then, there exists $u \in L$ such that $u \in w \amalg \Sigma^{*}$, which implies, for any $j \in\{1, \ldots, k\},\left|\pi_{j}(w)\right| \leqslant\left|\pi_{j}(u)\right|$. Set

$$
\left(f_{1}, \ldots, f_{k}\right)=\delta\left(s_{0}, u\right) \text { and }\left(s_{1}, \ldots, s_{k}\right)=\delta\left(s_{0}, w\right) .
$$

We define a state $t=\left(t_{1}, \ldots, t_{k}\right) \in S_{1} \times \ldots \times S_{k}$ as follows. Let $j \in\{1, \ldots, k\}$, then define $t_{j}$ according to the following cases:

(a) $f_{j}<i_{j}$. Then, as $\left|\pi_{j}(w)\right| \leqslant\left|\pi_{j}(u)\right|$ and $f_{j}<i_{j}$, which also implies $s_{j}<i_{j}$, we have, by the definition of the transition function of $\mathcal{C}_{L}$, then $s_{j} \leqslant f_{j}<i_{j}$. Set $t_{j}=f_{j}$.

(b) $i_{j} \leqslant f_{j}<i_{j}+p_{j}$. Set

$$
t_{j}=\left\{\begin{array}{l}
f_{j} \text { if } s_{j}<i_{j} ; \\
s_{j} \text { if } i_{j} \leqslant s_{j}<i_{j}+p_{j} .
\end{array}\right.
$$

Then, for any $j \in\{1, \ldots, k\}$,

$$
\left(\left(i_{j} \leqslant t_{j}<i_{j}+p_{j}\right) \wedge\left(i_{j} \leqslant f_{j}<i_{j}+p_{j}\right)\right) \text { or }\left(t_{j}=f_{j}\right) .
$$

Therefore, $t \in E^{\prime}$. By definition of $t$, we have $s \leqslant t$. So, we find $s \in F_{\downarrow}$. Hence $w \in L\left(\mathcal{A}_{\downarrow}\right)$. [End, Proof of the Claim]

3. Due to Equation (11), by switching the final state set of an automaton for $\downarrow\left(\Sigma^{*} \backslash L\right)$, we get a recognizing automaton for $\circlearrowleft L$. So, this is implied by the state complexity bound for $\downarrow L$.

4. By Equation (11), similarly as for $\circlearrowleft L$, the state complexity bound is implied.

So, all the state complexity bounds are established.

\section{Proofs for Section 4 (The Case of Commutative Group Languages)}

Theorem 11, Let $\Sigma=\left\{a_{1}, \ldots, a_{k}\right\}$ and $L \subseteq \Sigma^{*}$. Then, the following conditions are equivalent:

1. L is a commutative group language;

2. $L$ is a finite union of languages of the form $U_{1} \amalg \ldots \amalg U_{k}$ with $U_{j} \subseteq\left\{a_{j}\right\}^{*}$ a group language recognizable by an automaton with a single final state;

3. $L$ is a finite union of languages of the form $U_{1} \amalg \ldots \amalg U_{k}$ with $U_{j} \subseteq\left\{a_{j}\right\}^{*}$ being group languages.

Proof. Proof that (1) implies (2): Let $L \subseteq \Sigma^{*}$ be a commutative group language with recognizing commutative permutation automaton $\mathcal{A}=\left(\Sigma, Q, \delta, q_{0}, F\right)$. Then, for $q_{f} \in F$,

$$
L\left(\left(\Sigma, Q, \delta, q_{0},\left\{q_{f}\right\}\right)\right)=\bigsqcup_{j=1}^{k} \pi_{j}\left(L\left(\left(\Sigma, Q, \delta, q_{0},\left\{q_{f}\right\}\right)\right)\right) .
$$


This equation follows easily, the inclusion of the set on the left hand side in the set on the right hand side is true for any language. The other inclusion is implied for if we have a word in this language, then some permutation of it is in $L\left(\left(\Sigma, Q, \delta, q_{0},\left\{q_{f}\right\}\right)\right)$, but as the automaton is commutative the original automaton also ends up in the state $q_{f}$. By [Hof19, Corollary 9], the languages $\pi_{j}\left(L\left(\left(\Sigma, Q, \delta, q_{0},\left\{q_{f}\right\}\right)\right)\right)$ are unary group languages. These unary languages can be written as unions of languages recognizable by automata with a single final state. Then, as shuffle distributes over union, the claim follows.

Proof that (2) implies (3): This is clear.

Proof that (3) implies (1): By [Hof19, Lemma 13], the languages $U_{1} ш \ldots ш U_{k}$ are group languages and, as the $U_{j}$ are unary, these languages are commutative So, as the group languages and the commutative languages are closed under union [Pin97], we can deduce that $L$ is a commutative group language.

For lower bound results, we also need the next results.

Lemma 23. Let $\Sigma=\left\{a_{1}, \ldots, a_{k}\right\}$ and $\left(n_{1}, \ldots, n_{k}\right) \in \mathbb{N}_{0}^{k}$. Suppose for a commutative language $L \subseteq \Sigma^{*}$ we have

1. $\left\{\left.w \in \Sigma^{*}|\forall j \in\{1, \ldots, k\}:| w\right|_{a_{j}} \geqslant n_{j}\right\} \subseteq L$,

2. $\left\{\left.w \in \Sigma^{*}|\exists j \in\{1, \ldots, k\}:| w\right|_{a_{j}}=\min \left\{n_{j}-1,0\right\}\right\} \cap L=\varnothing$.

Then $\operatorname{sc}(L)=\prod_{j=1}^{k}\left(n_{j}+1\right)$ with index vector $\left(n_{1}, \ldots, n_{k}\right)$ and period vector $(1, \ldots, 1)$.

Proof. Set $\mathcal{C}=\left(\Sigma,\left[n_{1}+1\right] \times \ldots \times\left[n_{k}+1\right], \delta,(0, \ldots, 0), F\right)$ with

$$
\begin{aligned}
\delta\left(\left(i_{1}, \ldots, i_{j-1}, i_{j}, i_{j+1}, \ldots, i_{k}\right), a_{j}\right) & \\
& =\left(i_{1}, \ldots, i_{j-1},\left(i_{j}+1\right) \bmod \left(n_{j}+1\right), i_{j+1}, \ldots, i_{k}\right) .
\end{aligned}
$$

and $F=\left\{\left(\min \left\{|w|_{a_{1}}, n_{1}\right\}, \ldots, \min \left\{|w|_{a_{k}}, n_{k}\right\}\right) \mid w \in L\right\}$. Then $L(\mathcal{C})=L$. Let $\left(i_{1}, \ldots, i_{k}\right),\left(l_{1}, \ldots, l_{k}\right) \in\left[n_{1}+1\right] \times \ldots \times\left[n_{k}+1\right]$ be distinct. Suppose, without loss of generality, $i_{j}>l_{j}$ for some $j \in\{1, \ldots, k\}$. This implies $0 \leqslant l_{j}<n_{j}$. Then

$$
\delta\left(\left(l_{1}, \ldots, l_{k}\right), a_{1}^{n_{1}} \cdots a_{j-1}^{n_{j-1}} a_{j}^{n_{j}-1-l_{j}} a_{j+1}^{n_{j+1}} \cdots a_{k}^{n_{k}}\right) \notin F
$$

but

$$
\delta\left(\left(i_{1}, \ldots, i_{k}\right), a_{1}^{n_{1}} \cdots a_{j-1}^{n_{j-1}} a_{j}^{n_{j}-1-l_{j}} a_{j+1}^{n_{j+1}} \cdots a_{k}^{n_{k}}\right) \in F
$$

Hence, all states are distinguishable and $\mathcal{C}$ is isomorphic to the minimal automaton of $L$, which proves the claim.

Lemma 24. (Refining a result from [PSO2]) Let $\mathcal{A}=\left(\{a\}, Q, \delta, s_{0}, F\right)$ and $\mathcal{B}=$ $\left(\{a\}, P, \mu, t_{0}, E\right)$ be two unary automata with index zero and periods $p$ and $q$ respectively. Write $F=\left\{f_{1}, \ldots, f_{n}\right\}, E=\left\{e_{1}, \ldots, e_{m}\right\}$. Then $L(\mathcal{A}) L(\mathcal{B})$ could be accepted by an automaton $\mathcal{C}=\left(\{a\}, R, \eta, r_{0}, T\right)$ with index $\operatorname{lcm}(p, q)-1$, period $\operatorname{gcd}(p, q)$ and $T=\bigcup_{l=1}^{n} \bigcup_{h=1}^{m} T_{l, h}$ such that

$$
\left\{w \mid \eta\left(r_{0}, w\right) \in T_{l, h}\right\}=\left\{u \mid \delta\left(s_{0}, u\right)=f_{l}\right\} \cdot\left\{v \mid \mu\left(t_{0}, v\right)=e_{h}\right\}
$$


for $l \in\{1, \ldots, n\}$ and $h \in\{1, \ldots, m\}$. This result is optimal in the sense that there exists automata $\mathcal{A}$ and $\mathcal{B}$ as above such that $\mathcal{C}$ is isomorphic to the minimal automaton of $L(\mathcal{A}) L(\mathcal{B})$.

Proof. The existence and optimality was stated in [PS02] as Theorem 8, we only show the additional part about the final states. With the notation from the statement, set $\mathcal{A}_{l}=\left(\{a\}, Q, \delta, s_{0},\left\{f_{l}\right\}\right)$ and $\mathcal{B}_{h}=\left(\{a\}, Q, \mu, t_{0},\left\{e_{h}\right\}\right)$ for $l \in$ $\{1, \ldots, n\}$ and $h \in\{1, \ldots, m\}$. Then by [PS02] we have an automaton with index $\operatorname{lcm}(p, q)-1$ and period $\operatorname{gcd}(p, q)$ accepting $L\left(\mathcal{A}_{l}\right) \cdot L\left(\mathcal{B}_{h}\right)$ for $l \in\{1, \ldots, n\}$ and $h \in\{1, \ldots, m\}$. As the index and period determines the form of the automaton uniquely, we can suppose the only differing parts of those automata for each $l \in\{1, \ldots, n\}$ and $h \in\{1, \ldots, m\}$ are the final states. Hence, we can write $\mathcal{C}_{l, h}=$ $\left(\{a\}, R, \eta, r_{0}, T_{l, h}\right)$ where $R, \eta$ and the start state $r_{0}$ are independent of $l$ and $h$, and

$$
L\left(\left(\{a\}, R, \eta, r_{0}, T_{l, h}\right)\right)=L\left(\mathcal{A}_{l}\right) L\left(\mathcal{B}_{h}\right) .
$$

Set $T=\bigcup_{l=1}^{n} \bigcup_{h=1}^{m} T_{l, h}$, then

$$
\begin{aligned}
L\left(\left(\{a\}, R, \eta, r_{0}, T\right)\right) & =\bigcup_{l=1}^{n} \bigcup_{h=1}^{m} L\left(\mathcal{A}_{l}\right) L\left(\mathcal{B}_{h}\right) \\
& =\left(\bigcup_{l=1}^{n} L\left(\mathcal{A}_{l}\right)\right) \cdot\left(\bigcup_{h=1}^{m} L\left(\mathcal{B}_{h}\right)\right) \\
& =L(\mathcal{A}) L(\mathcal{B}) .
\end{aligned}
$$

This shows our claim.

Lemma 25. (Combining results from [Hof,Hof19]) Let $\Sigma=\left\{a_{1}, \ldots, a_{k}\right\}$ and $L \subseteq \Sigma^{*}$ be a commutative regular language with index vector $\left(i_{1}, \ldots, i_{k}\right)$ and period vector $\left(p_{1}, \ldots, p_{k}\right)$. Then we can write

$$
L=\bigcup_{l=1}^{n} U_{1}^{(l)} \amalg \ldots \amalg U_{k}^{(l)}
$$

with unary languages $U_{j}^{(l)} \subseteq\left\{a_{j}\right\}^{*}$ for $j \in\{1, \ldots, k\}$. Furthermore, we can find unary automata $\mathcal{A}_{j}=\left(\left\{a_{j}\right\}, Q_{j}, \delta_{j}, s_{j}, F_{j}\right)$ with indices $i_{j}$ and periods $p_{j}$ and with $F_{j}=\left\{f_{j}^{(1)}, \ldots, f_{j}^{(n)}\right\}$ such that for $l \in\{1, \ldots, n\}$

$$
U_{j}^{(l)}=\left\{u \in\left\{a_{j}\right\}^{*}: \delta_{j}(u)=f_{j}^{(l)}\right\}
$$

and $\left|Q_{j}\right|=i_{j}+p_{j}$. Hence $L\left(\mathcal{A}_{j}\right)=\left\{a_{j}^{|u|_{a_{j}}} \mid u \in L\right\}=\bigcup_{l=1}^{n} U_{j}^{l}$.

Proof. We refer to [Hof19,Hof,GA08] for the definition of the minimal commutative automaton. We also use the same notation for the languages $U_{j}^{(l)}$ as used in [Hof,Hof19]. The first claim is stated as Corollary 2 in [Hof19,Hof]. Lemma 5 of [Hof,Hof19] states that we can derive from the minimal commutative automaton a unary automaton with $i_{j}+p_{j}$ states such that $U_{j}^{(l)}$ for $l \in\{1, \ldots, n\}$ 
is precisely the set of words that lead this automaton into a single final state. For each $U_{j}^{(l)}$ the automaton with $i_{j}+p_{j}$ states is the same automaton. Hence, collecting in $F$ all the final states corresponding to the $U_{j}^{(l)}$ gives the claim. Note that we could have $U_{j}^{(l)}=U_{j}^{\left(l^{\prime}\right)}$ for distinct $l, l^{\prime} \in\{1, \ldots, n\}$. Also note that by definition, and as the minimal commutative automaton is deterministic, if $U_{j}^{(l)} \neq U_{j}^{\left(l^{\prime}\right)}$, then $U_{j}^{(l)} \cap U_{j}^{\left(l^{\prime}\right)}=\varnothing$.

Theorem 12, Let $\Sigma=\left\{a_{1}, \ldots, a_{k}\right\}$. For commutative group languages $U, V \subseteq$ $\Sigma^{*}$ with period vectors $\left(p_{1}, \ldots, p_{k}\right)$ and $\left(q_{1}, \ldots, q_{k}\right)$ their shuffle $U \amalg V$ has index vector $\left(i_{1}, \ldots, i_{k}\right)$ with $i_{j}=\operatorname{lcm}\left(p_{j}, q_{j}\right)-1$ for $j \in\{1, \ldots, k\}$ and period vector $\left(\operatorname{gcd}\left(p_{1}, q_{1}\right), \ldots, \operatorname{gcd}\left(p_{k}, q_{k}\right)\right)$. Hence, by Theorem 3 ,

$$
\operatorname{sc}(U \amalg V) \leqslant \prod_{j=1}^{k}\left(\operatorname{gcd}\left(p_{j}, q_{j}\right)+\operatorname{lcm}\left(p_{j}, q_{j}\right)-1\right) .
$$

Furthermore, there exist commutative group languages such that a minimal automaton recognizing their shuffle reaches the bound.

Proof. Write

$$
\begin{aligned}
& U=\bigcup_{l=1}^{n} U_{1}^{(l)} \amalg \ldots ш U_{k}^{(l)} \\
& V=\bigcup_{h=1}^{m} V_{1}^{(h)} \amalg \ldots ш V_{k}^{(h)}
\end{aligned}
$$

with, for $j \in\{1, \ldots, k\}$, unary automata $\mathcal{A}_{j}=\left(\left\{a_{j}\right\}, Q_{j}, \delta_{j}, s_{j}, F_{j}\right)$ and $\mathcal{B}_{j}=$ $\left(\left\{a_{j}\right\}, P_{j}, \mu_{j}, t_{j}, E_{j}\right), F_{j}=\left\{f_{j}^{(l)} \mid l \in\{1, \ldots, n\}\right\}$ and $E_{j}=\left\{e_{j}^{(h)} \mid h \in\{1, \ldots, m\}\right\}$, with indices zero and periods $p_{j}$ and $q_{j}$ respectively, according to Lemma 25, Let $\mathcal{C}_{j}=\left(\left\{a_{j}\right\}, R_{j}, \eta_{j}, r_{j}, T_{j}\right)$ with $T_{j}=\bigcup_{l=1}^{n} \bigcup_{h=1}^{m} T_{j}^{(l, h)}$ be automata according to Lemma 24 such that

$$
\left\{u \mid \eta_{j}\left(r_{j}, u\right) \in T_{j}^{(l, h)}\right\}=U_{j}^{(l)} \cdot V_{j}^{(h)}
$$

for $l \in\{1, \ldots, n\}$ and $h \in\{1, \ldots, m\}$. Hence, the indices and periods of these automata, as stated in Lemma 24, are precisely $\operatorname{lcm}\left(p_{j}, q_{j}\right)-1$ and $\operatorname{gcd}\left(p_{j}, q_{j}\right)$. Define

$$
\mathcal{C}=\left(\Sigma, R_{1} \times \ldots \times R_{k}, \eta,\left(r_{1}, \ldots, r_{k}\right), T\right)
$$

with

$$
T=\bigcup_{l=1}^{n} \bigcup_{h=1}^{m} T_{1}^{(l, h)} \times \ldots \times T_{k}^{(l, h)}
$$

and transition function $\delta\left(\left(r_{1}, \ldots, r_{j}, \ldots, r_{k}\right), a_{j}\right)=\left(r_{1}, \ldots, \delta_{j}\left(r_{j}, a_{j}\right), \ldots, r_{k}\right)$ for $\left(r_{1}, \ldots, r_{j}, \ldots, r_{k}\right) \in R_{1} \times \ldots \times R_{k}$. By construction of $\mathcal{C}$ and Equation (14), we have

$$
\delta\left(u,\left(r_{1}, \ldots, r_{k}\right)\right) \in T_{1}^{(l, h)} \times \ldots \times T_{k}^{(l, h)} \Leftrightarrow u \in\left(U_{1}^{(l)} V_{1}^{(h)}\right) ш \ldots ш\left(U_{k}^{(l)} V_{k}^{(h)}\right)
$$


for $l \in\{1, \ldots, n\}$ and $h \in\{1, \ldots, m\}$. Hence

$$
\begin{aligned}
& L(\mathcal{C})=\bigcup_{l=1}^{n} \bigcup_{h=1}^{m}\left(U_{1}^{(l)} V_{1}^{(h)}\right) ш \ldots ш\left(U_{k}^{(l)} V_{k}^{(h)}\right) \\
& =\bigcup_{l=1}^{n} \bigcup_{h=1}^{m} U_{1}^{(l)} \amalg \ldots ш U_{k}^{(l)} \amalg V_{1}^{(l)} \amalg \ldots ш V_{k}^{(l)} \\
& =\left(\bigcup_{l=1}^{n} U_{1}^{(l)} \uplus \ldots \uplus U_{k}^{(l)}\right) ш\left(\bigcup_{h=1}^{m} V_{1}^{(l)} \amalg \ldots ш V_{k}^{(l)}\right) \\
& =U \amalg V
\end{aligned}
$$

as the shuffle operation is commutative and distributive over union and for unary languages shuffle and concatenation are the same operations. Now, we show that the bound is sharp. Let $p, q$ be two distinct prime numbers. Then, every number greater than $p \cdot q-(p+q)+1$ could be written in the form $a p+b q$ with $a, b \geqslant 0$, and this is the minimal number with this property, i.e., $p q-(p+q)$ could not be written in that way; see [PS02]. Set $V_{j}=a_{j}^{p-1}\left(a_{j}^{p}\right)^{*}, W_{j}=a_{j}^{q-1}\left(a_{j}^{q}\right)^{*}$ and $U_{j}=\left\{a_{j}^{p+q-2+a p+b q} \mid a, b \geqslant 0\right\}=V_{j} W_{j}$. Then

$$
\operatorname{sc}\left(V_{1} ш \ldots ш V_{k}\right)=p^{k} .
$$

For, the permutation automaton $\mathcal{C}=\left(\Sigma,[p]^{k}, \delta,(0, \ldots, 0),\{(p-2, \ldots, p-2)\}\right)$ with

$$
\delta\left(\left(i_{1}, \ldots, i_{j-1}, i_{j}, i_{j+1}, \ldots, i_{k}\right), a_{j}\right)=\left(i_{1}, \ldots, i_{j-1},\left(i_{j}+1\right) \bmod p, i_{j+1}, \ldots, i_{k}\right)
$$

accepts $V_{1} \uplus \ldots ш V_{k}$, and it is easy to see that if a language is accepted by a permutation automaton with a single final state, then this automaton is minimal. Similarly, $\operatorname{sc}\left(W_{1} ш \ldots ш W_{k}\right)=q^{k}$.

Set $n_{j}=p q-1$ for $j \in\{1, \ldots, k\}$. Then the language

$$
\begin{aligned}
L=U_{1} ш \ldots ш U_{k}=\left(V_{1} W_{1}\right) & \ldots \\
& =\left(V_{1} W_{k}\right) \\
&
\end{aligned}
$$

fulfills the prerequisites of Lemma 23 with $\left(n_{1}, \ldots, n_{k}\right)$, as for each $j \in\{1, \ldots k\}$ we have $a_{j}^{p q-2} \notin U_{j}$ and $a_{j}^{p q-1} a_{j}^{*} \subseteq U_{j}$. Hence,

$$
\operatorname{sc}(L)=(p q)^{|\Sigma|}=(\operatorname{gcd}(p, q)+\operatorname{lcm}(p, q)-1)^{|\Sigma|}
$$

and the bound given by the statement is attained.

Proposition 14, Let $n, m>0$ be coprime numbers. Then, there exist commutative group languages of states complexities $n$ and $m$ such that their shuffle has state complexity $\mathrm{nm}$. 
Proof. Let $a \in \Sigma$ and $n, m>0$ be coprime numbers. Set $U=\left\{w \in \Sigma^{*} \mid\right.$ $\left.|w|_{a} \equiv n-1(\bmod n)\right\}=a^{n-1}\left(a^{n}\right)^{*} w(\Sigma \backslash\{a\})^{*}$ and $V=\left\{\left.w \in \Sigma^{*}|| w\right|_{a} \equiv\right.$ $m-1(\bmod m)\}=a^{m-1}\left(a^{m}\right) ш(\Sigma \backslash\{a\})^{*}$. Then, by a number-theoretical result from [YZS94, Lemma 5.1], $U \uplus V=F \amalg(\Sigma \backslash\{a\})^{*} \cup a^{n m-1} a^{*} \amalg(\Sigma \backslash\{a\})^{*}$ for some finite $F \subseteq \Sigma^{*}$ with $a^{n m-2} \notin F$ and this language has state complexity $n m$.

Proposition 15, Let $L \subseteq \Sigma^{*}$ be a commutative group language. Then the downward closure $\downarrow L$ equals $\Sigma^{*}$.

Proof. Let $\mathcal{A}=\left(\Sigma, Q, \delta, q_{0}, F\right)$ be a permutation automaton with $L(\mathcal{A})=L$. If $L=\{\varepsilon\}$, then the statement is clear. So, suppose $L \neq\{\varepsilon\}$. Note that $L \neq \varnothing$, as $\varnothing$ is never a group language. Let, without loss of generality, $\left\{\left.a|\exists w \in L:| w\right|_{a}>\right.$ $0\}^{*}=\left\{a_{1}, \ldots, a_{l}\right\}, 0<l \leqslant k$. Choose, for any $j \in\{1, \ldots, l\}$, a word $w_{j} \in L$ with $\left|w_{j}\right|_{a_{j}}>0$. Set $w=w_{1} \cdots w_{l}$. Then, some power $w^{m}$ acts as the identity on $Q$. Hence, $w_{1} w^{r \cdot m} \in L$ for any $r \geqslant 0$. Let $u \in\left\{a_{1}, \ldots, a_{l}\right\}^{*}$ be arbitrary, then, as

$$
\left|w_{1} w^{r \cdot m}\right|_{a_{j}}<\left|w_{1} w^{(r+1) \cdot m}\right|_{a_{j}}
$$

for any $r \geqslant 0$ and $j \in\{1, \ldots, l\}$, there exists $r \geqslant 0$ such that, for any $j \in\{1, \ldots, l\}$,

$$
|u|_{a_{j}}<\left|w_{1} w^{r \cdot m}\right|_{a_{j}} .
$$

But then, we can reorder the letters in $w_{1} w^{r \cdot m}$ such that $u$ is a scattered subsequence of this word, i.e., we can delete letters from this word such that $u$ results. More formally, we have $\left(u \amalg \Sigma^{*}\right) \cap \operatorname{perm}\left(w_{1} w^{r \cdot m}\right) \neq \varnothing$. As $L$ is commutative, $\operatorname{perm}\left(w_{1} w^{r \cdot m}\right) \subseteq L$ and we find $u \in \downarrow L$.

\section{References for the Appendix}

BS73. J. A. Brzozowski and I. Simon. Characterizations of locally testable events. Discrete Mathematics, 4(3):243-271, 1973.

FH19. Henning Fernau and Stefan Hoffmann. Extensions to minimal synchronizing words. J. Autom. Lang. Comb., 24(2-4):287-307, 2019.

GA08. A. Cano Gómez and G. I. Alvarez. Learning commutative regular languages. In Alexander Clark, François Coste, and Laurent Miclet, editors, ICGI 2008, volume 5278 of $L N C S$, pages 71-83. Springer, 2008.

Hof. S. Hoffmann. Commutative regular languages - properties and state complexity. Information and Computation. (to appear).

Hof19. Stefan Hoffmann. Commutative regular languages - properties and state complexity. In Miroslav Ciric, Manfred Droste, and Jean-Éric Pin, editors, CAI 2019, Niš, Serbia, June 30 - July 4, 2019, Proceedings, volume 11545 of LNCS, pages 151-163. Springer, 2019.

Pin97. Jean-Éric Pin. Syntactic semigroups. In Grzegorz Rozenberg and Arto Salomaa, editors, Handbook of Formal Languages, Volume 1, pages 679-746. Springer, 1997.

PS02. G. Pighizzini and J. Shallit. Unary language operations, state complexity and jacobsthal's function. International Journal of Foundations of Computer Science, 13(1):145-159, 2002. 
YZS94. S. Yu, Q. Zhuang, and K. Salomaa. The state complexities of some basic operations on regular languages. Theoretical Computer Science, 125(2):315328, March 1994. 